\title{
Recent Advances in Marching-on-in-Time Schemes for Solving Time Domain Volume Integral Equations
}

\author{
Sadeed B. Sayed ${ }^{(1)}$, Huseyin A. Ulku ${ }^{(1,2)}$, and Hakan Bagci ${ }^{*(1)}$ \\ (1) Division of Computer, Electrical, and Mathematical Sciences and Engineering \\ King Abdullah University of Science and Technology (KAUST), Thuwal, Saudi Arabia \\ (2) Department of Electronics Engineering \\ Gebze Institute of Technology, Kocaeli, Turkey
}

Transient electromagnetic field interactions on inhomogeneous penetrable scatterers can be analyzed by solving time domain volume integral equations (TDVIEs). TDVIEs are constructed by setting the summation of the incident and scattered field intensities to the total field intensity on the volumetric support of the scatterer. The unknown can be the field intensity or flux/current density. Representing the total field intensity in terms of the unknown using the relevant constitutive relation and the scattered field intensity in terms of the spatiotemporal convolution of the unknown with the Green function yield the final form of the TDVIE. The unknown is expanded in terms of local spatial and temporal basis functions. Inserting this expansion into the TDVIE and testing the resulting equation at discrete times yield a system of equations that is solved by the marching on-in-time (MOT) scheme. At each time step, a smaller system of equations, termed MOT system is solved for the coefficients of the expansion. The right-hand side of this system consists of the tested incident field and discretized spatio-temporal convolution of the unknown samples computed at the previous time steps with the Green function.

Unlike finite difference and finite element schemes, MOT-TDVIE solvers require discretization of only the scatterers, do not call for artificial absorbing boundary conditions, and are more robust to numerical phase dispersion. On the other hand, their computational cost is high, they tend to become unstable when applied to high-contrast scatterers, and the implicit nature of time marching makes incorporation of nonlinear constitutive relations more difficult. Development of plane wave time domain (PWTD) and FFT-based schemes has significantly reduced the computational cost of the MOT-TDVIE solvers. But the other two challenges are yet to be addressed.

This presentation will describe two new MOT techniques developed to address these challenges. (i) MOT-TDVIE solver high-contrast scatterers. The stability is achieved using two-sided (non-causal) temporal basis functions. The causality of the MOT scheme is restored using an extrapolation scheme, which is specifically trained using oscillating and exponentially decaying functions. Consequently, the resulting MOT scheme is highly accurate in computing (oscillating and exponentially decaying) fields excited inside the high-contrast scatterer. (ii) MOT-TDVIE solver for nonlinear scatterers. Both flux density and field intensity are kept as unknowns and the nonlinear constitutive relation and the TDVIE are discretized separately. The resulting coupled system is integrated in time to compute the samples of the unknowns using an explicit multistep scheme. Explicitness allows for straightforward incorporation of the nonlinearity as a function call on the right hand side of the coupled system of equations.

Numerical results, which demonstrate the accuracy, stability, and applicability of the MOT schemes described above, will be presented. 